\title{
La sociabilidad: una vivencia en diferentes espacios de aprendizaje
}

\section{Sociability: an experience in different learning spaces}

\section{A sociabilidade: uma vivência em diferentes espaços de aprendizagem}

\author{
Pedro Elías Ramírez Bustos \\ Doctor en Historia y Política Moderna \\ Universidad Pablo de Olavide de Sevilla \\ pramirez6@udi.edu.co
}

\section{Socorro Astrid Portilla Castellanos}

Doctora en Educación

Universidad de la Salle de Costa Rica

sportilla2@udi.edu.co

\section{Resumen}

El presente artículo de reflexión hace parte de la fase I, del proyecto de investigación Caracterización de las formas de sociabilidad de los estudiantes de la UDI, cuyo propósito es determinar cómo se socializan los estudiantes en los ámbitos educativos terciarios, su interacción con los diferentes entornos y los efectos en su proceso educativo. Durante esta fase se construye el estado del arte, aspecto fundamental para la interpretación de la información y el diseño de una propuesta de intervención que se espera construir al finalizar el proyecto.

La propuesta se enmarca dentro de la base teórica de autores internacionales como, (Giddens, 2001), (Bourdieu, 2008) , (Bauman, 2001); historiadores de primer grado como Agulhon, Guerra y Furet; filósofos como Habermas, (Habermas, 1987) Gadamer, y teóricos de la pedagogía crítica como, (Girox, 1998), (Freire, 2005) acompañados por autores nacionales entre los cuales se encuentran los sociólogos Celis Giraldo, Guatame \& Castro (Celis Giraldo \& Guatame Castro, 2019), que han reflexionado sobre la asociatividad por la ruta de la historia de la sociabilidad y que aborda los instrumentos que permiten comprender el problema de investigación en torno a la necesidad de entender los nuevos espacios de relaciones, determinar sus formas y vínculos, dentro de un ámbito concreto, como lo es la Universidad de Investigación y Desarrollo UDI (Sede Bucaramanga).

Palabras claves: Sociabilidad, vínculos, ambientes educativos, interacción, nichos de aprendizaje. 


\begin{abstract}
This article of reflection is part of phase I, of the research project Characterization of the forms of sociability of the students of the UDI, whose purpose is to determine how students socialize in tertiary educational studies, their interaction with different environments and the effects on their educational process. During this phase the state of the art is constructed, a fundamental aspect for the interpretation of the information and the design of an intervention proposal that is expected to be built at the end of the project.
\end{abstract}

The proposal is framed within the theoretical basis of international authors such as Giddens, Bourdieu, Bauman; First-degree historians such as Agulhon, Guerra and Furet; philosophers such as Habermas, Gadamer and critics of critical pedagogy such as Giroux, Freire, accompanied by national authors among whom are the sociologists Celis Giraldo, Guatame Castro who have reflected on the associativity along the path of the history of sociability and which addresses the instruments that allow us to understand the research problem around the need to understand the new spaces of relationships, determine their forms and links, within a specific area, such as the University of Research and Development UDI (Bucaramanga Headquarters).

Keywords: Sociability, links, educational environments, interaction, learning niches

\title{
Resumo
}

O presente artigo de reflexão faz parte da fase I do projeto de pesquisa Caracterização das formas de sociabilidade dos alunos da UDI. O objetivo é determinar como se socializam os alunos nos ambientes educativos terciários, sua interação com os diferentes ambientes e os efeitos em seu processo educacional. Nesta fase constrói-se o estado da arte, importante na interpretação da informação e na consecução de uma proposta de intervenção que se espera construir ao finalizar o projeto.

A proposta se enquadra na base teórica de autores internacionais como: (Giddens, 2001), (Bourdieu, 2008), (Bauman, 2001); historiadores principais como: Agulhon, Guerra y Furet; filósofos como: Habermas, (Habermas, 1987) Gadamer, e teóricos da pedagogia crítica como: (Girox, 1998), (Freire, 2005 ) junto a autores nacionais entre o quais encontrase os sociólogos Celis Giraldo, Guatame \& Castro (Celis Giraldo \& Guatame Castro, 2019), eles têm refletido sobre la associatividade pelo caminho da história da sociabilidade e abordam os instrumentos que permitem compreender o problema de pesquisa ao redor da necessidade de entender os novos espaços de relacionamento, determinar suas formas e vínculos num ambiente concreto como a Universidad de Investigación y Deasrrollo UDI (sede da cidade de Bucaramanga).

Palavras-chave: Sociabilidade, vínculos, ambientes educacionais, interação, nicho de aprendizagem.

\section{Introducción}

En el marco de la sociabilidad la intersubjetividad producida por la vida asociativa insta a conocer los diferentes ámbitos y espacios de la interacción entre los individuos, las razones de su identidad y pertenencia a los grupos y así mismo indagar por las formas como los estudiantes de la Universidad de Investigación y Desarrollo conciben y viven las relaciones interpersonales o la sociabilidad.

Hoy reconocemos dimensiones en las experiencias relacionales cotidianas producidas en la casa, calle y universidad, fabricados por fenómenos relacionados con la Globalización (redes sociales, conciencia ambiental y desnacionalización), la política neoliberal 
(flexibilidad, individualismo y desarrollo sostenible), la posmodernidad (multiculturalismo, hibridación, rechazo de lo moderno). Todo esto, sumado, a la experiencia histórica de la ciudad y la nación.

Estas transformaciones recién abiertas permiten prestar a su vez gran atención al tipo de lazo social, las diferentes formas y ámbitos de sociabilidad dentro de espacios singulares de la ciudad, como los que pueden encontrarse a los alrededores de la Universidad de Investigación y Desarrollo UDI y al interior de sus propias instalaciones, lo que hace posible identificar dimensiones hasta ahora ignoradas y, menos aún, codificadas en sus distintas y profundas formas.

Reconocidos sociólogos
como Giddens, Bourdieu,
cuestionado el concepto de "Globalineos hación
Económica" y mostrado sus consecuencias sobre la sociedad y el Estado de Bienestar. Historiadores de primer grado como Maurice Agulhon, François Xavier-Guerra y François Furet, por mencionar los que eligieron llevar la historia política por la ruta de la historia de las sociabilidades con el fin de explicar los cambios de la política en la segunda mitad del siglo XVIII y el tránsito como sociedad moderna en el siglo XIX, se diferencian de los primeros en que ellos y sus discípulos más sobresalientes "estudian el advenimiento de la era democrática" (González, 2008). Bauman, Bourdieu y Giddens, como científicos sociales, se interesan por el cambio social y las transformaciones históricas de las sociedades en el presente, pasando por alto el concepto de sociabilidad en sus lecturas especialmente de las dos últimas décadas del siglo XX y las primeras del siglo XXI. Es innegable que puede, e incluso, debe añadirse que entre ellos hay un punto de contacto: unos y otros se han interesado en los procesos de cambio y transformación de la sociedad contemporánea o moderna. Sin embargo, el problema radica en que nuestro presente se refleja como un tiempo que tiene muy poco en común, con las formas de sociabilidad política, con las que se dio inició a la modernidad política en Iberoamérica durante el siglo XIX, en consecuencia, a la sociabilidad moderna en los países latinoamericanos. Según Bauman, por decirlo así, en este momento:

[... los espacios urbanos donde los ocupantes de las diversas zonas residenciales puedan encontrarse cara a cara, hablar de bueyes perdidos, abordarse y desafiarse, conversar, reñir, discrepar o coincidir, elevar sus problemas particulares al nivel de asuntos públicos y hacer de éstos materia de interés particular -las ágoras "privadas/públicas" de Cornelius Castoriadis- disminuyen rápidamente en dimensiones y número. Los pocos que quedan tienden a ser cada vez más selectivos: refuerzan, en lugar de reparar, los daños causados por las fuerzas desintegradoras (Bauman, 2001).

En las circunstancias presentes, estas diferentes formas de integrarse y distinguirse en sociedad, que se inscribe en una red de fenómenos a "escala global", permiten que los lazos y el nuevo espacio de relaciones, que afectan el ámbito de determinados grupos de la Universidad de Investigación y Desarrollo UDI, serán cada vez más difíciles de caracterizar, conforme a los análisis establecidos por la nueva historia política "para la comprensión del siglo XIX, las mutaciones de la sociabilidad y las prácticas sociales, el nacimiento de la política moderna y la construcción de la nación" (González, 2008), han sido determinantes en las formas en que los jóvenes interactúan en los diferentes nichos de aprendizaje con los que desarrollan vínculos afectivos, familiares, académicos, sociales.

Con esta investigación se pretende ampliar el modelo teórico de las formas de sociabilidad, es decir, aspiramos con este trabajo a extender su clasificación (sociabilidad política, sociabilidad democrática, sociabilidad rural o urbana, sociabilidad lectora, etcétera), distinguiendo otros modos de sociabilidad que rodean las disposiciones sociales y prácticas en que se integran los actores sociales en la UDI. 
El interés del grupo se centra en las formas de sociabilidad y sus transformaciones que ciertamente están relacionadas, con los profundos cambios generados a partir de los años 80 . Estas tendencias que hacen parte de procesos de amplio espectro como la globalización económica y cultural, se expresan también en el plano individual; la juventud al instaurar un nuevo espacio de relaciones borra cada vez más las formas de sociabilidad propias de la modernidad política, favorecidos por el constante uso de las nuevas tecnologías, la pérdida del territorio o barrio como epicentro del mundo y la puesta en marcha de la política neoliberal; como diría Bourdieu: "con su afán de transformar a cada agente social en un pequeño empresario de su propia vida, responsable de su éxito, pero también de su fracaso, de su miseria económica, cultural y simbólica" (Bourdieu, 2008).

El estado del arte de la investigación se fundamenta en indagaciones sobre la sociabilidad, desde distintos enfoques que han permitido avanzar en la comprensión y elaboración del concepto desde ámbitos o sectores en donde se aplica como patrón fundamental de las dinámicas propias de los distintos grupos sociales que se conforman en nuestra comunidad.

En el ámbito internacional, se encuentran trabajos relacionados con la sociabilidad en la Internet, las redes sociales y el multiculturalismo, entre otros. En este sentido, se puede resaltar la investigación realizada por Baquerin $\mathrm{y}$ Borgaminck (2013). Dicho estudio tuvo como propósitos explicar cómo internet es una nueva tecnología que no tiene una difusión homogénea en la estructura social y por lo tanto, podría profundizar las diferencias entre los actores sociales haciendo que algunos tengan una percepción del tiempo y del espacio diferente y desarrollen nuevas habilidades comunicativas y otros no, así como indagar si también se puede hablar de "tecnorricos" y "tecnopobres" entre jóvenes que, habiendo alcanzado un mismo grado de educación formal, no han desarrollado iguales habilidades comunicativas (Baquerin y Borgaminck, 2013). Las autoras expresan que su trabajo enuncia un paradigma de progreso según el cual la difusión de una nueva tecnología se interpreta como una alternativa de desarrollo social equitativo.

Sin embargo, acceder o no acceder a internet marca la primera brecha excluyendo del "progreso" a una parte importante de jóvenes que estructuralmente no reúne las condiciones socioeconómicas para participar de esta tecnología. La segunda brecha se establece entre los jóvenes que acceden en casa y los que acceden en el colegio y una tercera brecha se fija por el grado de motivación de los jóvenes que acceden a internet en casa. Estas brechas los diferencian a partir de conectarse o no, de la proximidad de la conexión y del tiempo de conexión.

En el segmento de los jóvenes que acceden a internet en casa, la variable motivación representa un efecto directo sobre el uso de internet mientras que el clima educativo del hogar ejerce un efecto indirecto significativo. Esta combinación eventual entre la motivación y el clima educativo del hogar se acerca al modelo de explicación contingente de Kwak, en la medida en que ambas variables explican, con diferente intensidad, el fenómeno del uso de esta tecnología.

Otro tipo de brecha se relaciona con las funciones disponibles en internet y las competencias necesarias para operarlas. Algunos jóvenes utilizan internet unilateralmente y otros desarrollan nuevas formas de conversación. En la exclusión del medio, de aquellos que no tienen acceso, en los distintos grados de proximidad física con el medio; en las distintas competencias comunicativas que suponen diferentes usos para diferentes objetivos, se establecen brechas explicadas, algunas por situaciones estructurales y otras por diferencias individuales. Por lo tanto, esta investigación muestra que "internet es una nueva tecnología que puede profundizar las diferencias entre los 
jóvenes, haciendo que algunos desarrollen nuevas habilidades comunicativas y otros no". (Baquerin y Borgaminck, 2013, pág. 114)

A partir de este estudio se precisó con los estudiantes en la UDI, la relevancia que estos dan a la forma de relacionarse, prefiriendo en un $87,6 \%$ las relaciones presenciales a las virtuales o al uso de las tecnologías para realizar trabajos académicos, jugar o interactuar.

Un referente en el contexto nacional es la investigación realizada por el Grupo de Investigación Protección Social y Conflicto de la Facultad de Derecho de la Universidad Libre de Bogotá, a través de su proyecto titulado "La protección social y sus características en Colombia" que fue liderado por el director del grupo, el Doctor en derecho y sociología, Francisco Rafael Ostau De Lafont. Dicho proyecto se formuló como problema de investigación la posibilidad de

determinar si la sociabilidad y la solidaridad del ser humano es connatural a él o parte de la cultura de su entorno, la respuesta está dada en que la sociabilidad y la solidaridad del ser humano es parte de su entorno cultural y por lo tanto su elemento esencial es el reconocimiento del otro a partir de la solidaridad. (Ostau de Lafont, 2009)

Los resultados que se logran evidenciar del estudio resaltan que

las personas son diferentes en forma innata, podría darse paso a la legitimación de la opresión y la discriminación. Pero si, por el contrario, se supone que la sociabilidad es connatural al hombre, ello indica que no es necesario desarrollar organizaciones sociales, puesto que la condición de sociabilidad hará parte constitutiva del genoma humano y la voluntad del ser humano de asociarse desaparecería. (Ostau de Lafont, 2009)
También se hace alusión a que el hombre es un ser social, y desde este punto de vista es imposible un hombre fuera de la sociedad, pues es precisamente el papel de lo social lo que le da la dimensión de hombre. Se concluye del estudio que el alto porcentaje de la negación de la población colombiana a la sociabilidad y a la solidaridad obedece a que dentro de la cultura colombiana hay un agente extraño que niega la existencia del otro, el otro como diferente y el otro que necesita ser reconocido en su relación social.

Otro importante trabajo es el realizado por Gilberto Loaiza Cano, desarrollado por Olave Quintero (2012). En este se realiza una reseña donde resalta los aspectos relevantes de la obra, sintetiza las partes que la componen y comenta los vacíos historiográficos que identifica el autor en su trabajo. Para Olave (2012),

el libro es el resultado de la aplicación de la noción de sociabilidad para construir una visión en conjunto de lo que fue el mundo asociativo durante el siglo XIX en Colombia. La investigación abarca un largo periodo que va de 1820 a 1886 , aunque en algunos momentos hace referencia a hechos ocurridos en el decenio de 1810 que son de vital importancia para entender los procesos que se dieron en las décadas siguientes. Por lo tanto, como bien lo indica el autor, este libro es una historia de los que fue el diverso mundo asociativo durante el siglo XIX colombiano.

Se resalta además que para Loaiza

la noción de sociabilidad empezó a ser utilizada en la historiografía universitaria a principios de 1990, pero no logró tener avances significativos para explicar la historia política del siglo XIX en el país. Por consiguiente, la noción sólo ha sido utilizada por algunos investigadores para explicar coyunturas, pero nunca para intentar crear una visión en conjunto del amplio mundo asociativo en el siglo XIX. (Olave,Quintero, 2012) 
Siendo lo anterior, precisamente, la novedad que se puede encontrar en la obra que presenta el profesor Loaiza.

El libro Sociabilidad, religión y política en la definición de la nación indica los antecedentes de cada práctica asociativa y el análisis se realiza a partir del diálogo que se establece entre la historiografía latinoamericana y los aportes que sobre el tema ha realizado la historiografía colombiana. Además, en él se evidencian los vacíos historiográficos que el autor logró identificar luego de realizar una amplia revisión con este libro de la historiografía colombiana en torno al estudio de la cultura política del siglo XIX. Vacíos que terminan convirtiéndose en invitaciones para futuras investigaciones que son de vital importancia para reconstruir la historia política y cultura del siglo XIX en Colombia (Olave, Quintero, 2012).

En el contexto local se puede encontrar el aporte hecho por (Duque Castro, 2010), en su texto Instituciones, organizaciones y familias. Bucaramanga, Colombia, 1857-1885, en el capítulo donde examina los "Vínculos y relaciones diversas al interior de las redes de sociabilidad de Bucaramanga" resalta que los vínculos comerciales, de parentesco y de amistad que unieron y dieron forma a las redes de sociabilidad fueron reforzados a través de estrechas alianzas "espirituales" y/o de "clientelismo" que se materializaron de diversas maneras. En algunas ocasiones esas alianzas fueron fruto del apadrinamiento en los matrimonios, $y$ en otras ocasiones de la promoción y ayuda para conseguir un cargo público dentro del aparato político o burocrático del Estado. Con respecto al tema del apadrinamiento matrimonial, los integrantes de las redes siempre procuraron que los testigos de sus matrimonios fueran sus socios, parientes o amigos cercanos, preferiblemente miembros de la red. Una importante idea que se puede resaltar en dicho estudio de la sociabilidad en Bucaramanga es la evidencia de

los vínculos comerciales, espirituales, familiares y de parentesco, materializados a través del apadrinamiento, el matrimonio, los negocios o la contratación de apoderados y dependientes de almacenes, propiciaron, acercaron y dieron forma al núcleo de la red de sociabilidad compuesta por familiares, parientes, socios y amigos. (Duque Castro, 2010)

En torno a la Sociabilidad como concepto, es necesario decir que la noción de sociabilidad fue extraída de su origen sociológico para ser de uso frecuente y equívoco en la historiografía contemporánea, sobre todo en la francesa. Hasta ahora no hay una clara definición del término, pero las precisiones han sido, como es frecuente, una consecuencia del acumulado de trabajos relacionados con el tema.

El historiador francés Maurice Agulhon es quizás el autor más reconocido en el estudio de formas de sociabilidad y es también quien expone de manera más dramática las vaguedades en la definición de su objeto de estudio. Sus estudios sobre la sociabilidad iniciaron desde la década de 1960, y sus esfuerzos por definir este concepto datan de un par de décadas después. Muchas veces, la naturaleza de los trabajos históricos aporta nuevos significados a las definiciones con que el autor intenta sintetizar el concepto que lo ha guiado en sus análisis. En 1981, Agulhon aventuró esta definición: "la historia de la sociabilidad es un poco la historia conjunta de la vida cotidiana, íntimamente ligada a aquella de la sicología colectiva" (Duque Castro, 2010).

La noción de sociabilidad es uno los puntos nodales en torno al cual se pueden organizar los variados discursos (sociales, políticos, educacionales, religiosos).

La "sociabilidad" es, por una parte, un concepto utilizado ampliamente en los discursos del siglo XIX y del XX tanto en Colombia como en el resto de América, es decir, es una categoría analítica que opera en la auto-comprensión de los procesos sociales que, especialmente, los sectores 
de elite y también los subalternos, utilizan en la época; por otra parte, aun aludiendo a las formas voluntarias de asociación que caracterizan conceptos análogos como el de "sociedad civil" o "esfera pública" en la literatura política europea, el concepto de sociabilidad alude a formas no exclusivamente burguesas (se puede hablar de sociabilidad popular, por ejemplo), desde las cuales es posible distinguir entre sociabilidades formales e informales, en las que adquiere un énfasis semántico particular en el carácter de "competencia" o "habilidad" adquirible en la práctica de la vida cotidiana.

Los estudios de las formas de sociabilidad hicieron necesaria una indagación sobre la intensidad y variaciones en las que se concreta el ideal moderno de las prácticas asociativas en Iberoamérica. A la luz de estas transformaciones de comienzo del siglo XIX y de las recientes formas de integrarse las comunidades, se reconoce la importancia de continuar explorando cómo fueron especializándose las formas de asociación según los propósitos de los asociados; en qué formas de asociación hubo acercamiento entre las élites y sectores más marginales de la vida pública. También es necesario preguntarse por las modalidades asociativas adoptadas por la Iglesia católica y los regímenes conservadores y liberales de nuestras sociedades latinoamericanas en el siglo XIX y XX.

En definitiva, la categoría de sociabilidad tiene una gran variedad de manifestaciones, entre las que se cuentan: Los partidos políticos, la masonería, las sociedades literarias, las sociedades católicas, las sociedades democráticas y los clubes literarios entre otros. Esta diversa gama de expresiones deja claro que, una vez el cientista social se ha decidido al uso de esta categoría analítica, se enfrenta a un tema realmente complejo que merece estudios detallados y un denodado rigor teóricometodológico.

Resulta importante aclarar que las formas de sociabilidad modernas parecen resumir la consciencia de grupos de individuos que encuentran en la asociación la posibilidad de conseguir más fácilmente sus propósitos, pero que también creen que con la fundación de sociedades alejan a aquellos individuos que les son despreciables.

En Colombia, en la línea de estudios de Sociabilidad política, un hito sociológico e historiográfico lo constituye la obra de (Guillen,Martinez, 1979) el autor, sustrayéndose por completo de la historiografía tradicional, logra con claridad mostrar el itinerario de las formas de poder desde la colonia hasta nuestros días, haciendo énfasis en las llamadas estructuras asociativas (Formas de Sociabilidad), y la organización de los grupos sociales. En su análisis se muestran tres modelos: El de la Encomienda que gobierna la vida colombiana hasta mediados del siglo XIX, La Hacienda, modelo que se desarrolla a partir de $1854 \mathrm{y}$ que se extiende hasta bien entrado el siglo XX, y el modelo de la Empresa, forma de Sociabilidad inspirada en las exigencias hechas por el modelo capitalista desde comienzos de siglo XX; según el autor, estos tres tipos asociativos son significativos para entender cómo se ha configurado históricamente el Poder en Colombia.

Una de las tesis centrales, hilo conductor además del discurso, es que para entender la política nacional es fundamental entender la dinámica propia de los partidos; se advierte en esta tesis que en buena medida la cultura política colombiana se encuentra permeada por las formas de sociabilidad generadas a partir del modelo hacendatario.

En el entorno de la UDI, los jóvenes participantes del proyecto, en esta primera fase manifiestan no estar interesados en temas relacionados con la política y la religión, aspectos considerados en épocas pasadas de vital importancia en la formación y consolidación de vínculos de sociabilidad, hoy en la población objeto de estudio, de poca relevancia, en contravía con las opiniones de los gestores del 
proyecto, los cuales consideran la política un aspecto pertinente para la comprensión de nuestra realidad nacional el esclarecimiento de los ámbitos del universo de las formas de sociabilidad, hecho que ayudaría significantemente a entender las distintas formas de violencia, los altos niveles de corrupción política y en general las profundas vicisitudes y sesgos que hemos tenido en la construcción de nuestro Estado-Nación. Por lo tanto, hay en esta categoría analítica un gran futuro investigativo que implica serios estudios de las interacciones entre varios actores: partidos políticos, fuerzas armadas, actores armados, la Iglesia, los medios de comunicación (prensa), los centros educativos, la familia, los intelectuales, los gremios, los grupos de presión, etc., y como elemento orientador, las ideologías para con ello lograr entender qué realmente somos.

La educación superior está llamada a ser el eslabón que posibilite fortalecer los vínculos sociales en los que interactúan los jóvenes y reconozcan la importancia de participar de manera activa en los rumbos orientadores de la sociabilidad del país, empezando por los entornos en que se relaciona y los temas que al interior de estos nichos de aprendizaje se manejan.

\section{Referencias}

Baquerin y Borgaminck. (2013). ¿Internet un medio de sociabilidad o exclusión? Comunicación y sociedad, versión on line, 95-118.

Bauman, Z. (2001). La Globalización consecuencias humanas. México: Fondo de Cultura Económica.

Bourdieu, P. (2008). Una invitación a la sociología reflexiva. Buenos Aires: Siglo XXI.

Celis Giraldo, J., \& Guatame Castro, P. (23 de 10 de 2019). La dinámica institucional, la sociabilidad y las prácticas escolares: unacercamiento metodológico desde la sociología. Obtenido de Revista Colombiana de Sociología 21: https://revistas.unal.edu.co/index.php/recs/issu e/view/1270

Duque, M. (11 de noviembre de 2010). Tesis doctorales Universidad de Barcelona. Obtenido de http://www.ub.edu/gtr/publicacions/mem10 11/Recerca/RD4.htm

Freire, P. (2005). A la sombra de este árbol. Barcelona: El Roure.

Giddens, A. (2001). Un mundo desbocado. Los efectos de la globalización en nuestras vidad. Madrid: Taurus.

Girox, H. (1998). La pedagogía de frontera en la era del posmodernismo y educación. Bogotá: De alba.

González, P. (2008). Civilidad y Política en los origenes de la nación Argentina. Buenos Aires: FCE.

Guillen,Martinez, F. (1979). El poder político en Colombia. Bogotá: Punta de lanza.

Habermas, J. (1987). La Teoria de la acción comunicativa I. Racionalidad de acción y racionalización socil. Buenos Aires: Taurus.

Olave,Quintero, V. (2012). Sociabilidad,religión y política en la definición de la nación colombia 1820-1886 de Gilberto Loaiza Cano. Rehmlac, 259 .

Ostau de Lafont, R. (2009). La sociabilidad y la solidaridad como elementos culturales de la protección social. Dialogos de saberes, 26.

Olave, V. (2012). "Sociabilidad, religión y política en la definición de la nación.Colombia, 1820-1886 de Gilberto Loaiza Cano. Revista REHMLAC, 93. 


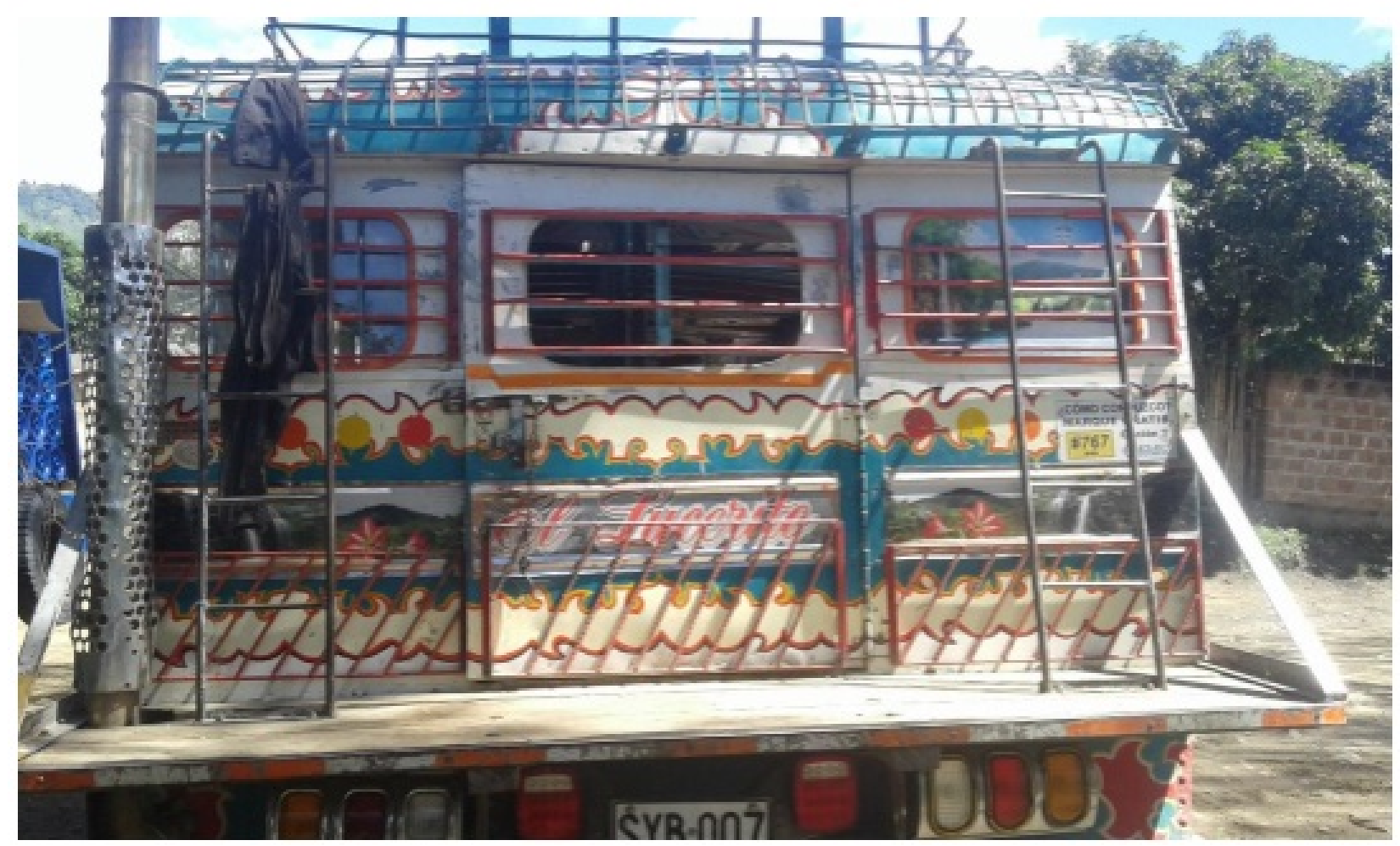

Número de la obra: 81

Título: Chiva "El Lucerito"

Autora: Margarita Chávarro Castro

Lugar: Municipio La Plata, Huila

Fecha de captura: Abril de 2013

Técnica: Fotografía digital

Fuente: Colección Margarita Chávarro Castro 